\title{
Experimental Techniques for Observing Dislocations by the Berg-Barrett Method
}

\author{
By A.P.L.Turner, T.VReeland, JR. And D.P. Pope \\ W.M. Keck Laboratory of Engineering Materials, California Institute of Technology, Pasadena, California, U.S.A.
}

\author{
(Received 30 October 1967)
}

\begin{abstract}
Experimental problems in the application of the Berg-Barrett Method to the observation of dislocations in metal single crystals are discussed. The problem of background noise caused by fluorescence from the specimen and inelastic scattering is considered. A criterion for selecting an appropriate filter to discriminate against the background noise is presented. The problem of multiple images is discussed and a scheme for selecting diffraction geometry to eliminate unwanted images is presented. A detailed description of the relatively simple equipment used in Berg-Barrett work is given.
\end{abstract}

\section{Introduction}

The Berg-Barrett X-ray extinction contrast (B-B) method of dislocation observation has proven to be a useful research tool. The method has been used in the W. M. Keck Laboratory to reveal individual dislocations in studies of dislocation mobility in several materials. In the course of these studies, various techniques have been developed which greatly simplify the application of the method to different materials with various crystal structures, using appropriate radiations and observation surfaces.

There are numerous reports (Roberts \& Gow, 1958; Newkirk, 1959; Lommel \& Kronberg, 1962; Robinson \& Levinstein, 1962; Webb, 1962; Schultz \& Armstrong, 1964; Armstrong, 1965; Austerman, Newkirk \& Smith, 1965; Pope, Vreeland \& Wood, 1967; Austerman \& Newkirk, 1967; Bonse, Hart \& Newkirk, 1967) in the literature of the application of the B-B method. However, poor experimental techniques have led some investigators to erroneous conclusions regarding the possibility of observing dislocations in some materials by means of the B-B method. Through work on a variety of materials the authors have developed certain experimental techniques which appear to be generally applicable for overcoming the difficulties of using the B-B method to observe dislocations.

\section{The method}

The arrangement of the experimental apparatus is shown schematically in Fig. 1. Radiation from the $\mathrm{X}$-ray source $A$ is incident on a surface (observation surface) of the specimen $B$. The angle $\varphi$ is adjusted so that the $K \alpha$ characteristic radiation of the X-ray tube is diffracted from a set of lattice planes (indicated by dotted lines) to produce an image $(a b)$ on the film $C$. The slits $D$ prevent the incident beam from exposing the film where the diffracted image appears.

Dislocations which lie close to the observation surface of the specimen are observed because they produce a line of contrast in the diffracted image. The nature and origin of this contrast is discussed by Newkirk,
Bonse \& Hart (1967). The strength of the contrast depends upon the relationships between the Burgers vector, $\mathbf{b}$, the diffraction vector, $\mathbf{g}$, and the slip plane normal n (Newkirk, Bonse \& Hart, 1967). This property of the contrast allows one to determine the direction of the Burgers vector (Newkirk, 1959).

\section{Problems}

\section{Signal to noise ratio}

The greatest problem in the application of the B-B method to the observation of dislocations in metals is background noise. By noise we mean radiation which reaches the film but is not part of the diffracted X-ray beam which we wish to observe. There are three sources of noise: fluorescent radiation from the specimen, inelastically scattered X-rays, and additional diffracted beams. Noise of the first two types produces a nearly uniform blackening of the film superimposed on the image. When the Bragg condition is satisfied for more than one set of lattice planes multiple images are produced on the film. These images are displaced relative to each other so that contrast effects are obscured. It has been found that in some metallic crystals the contrast at dislocations in the B-B image is nearly impossible to detect unless steps are taken to reduce the intensity of the background noise which reaches the film.

\section{Fluorescence and inelastic scattering}

The difficulty presented by fluorescent radiation from the specimen depends strongly on the relative wavelengths of the fluorescence and the diffracted beam.



Fig. 1. Schematic diagram of the B-B method. A divergent beam of X-rays originates from $\mathrm{X}$-ray source $A$, is diffracted from the observation surface of specimen $B$ forming an image $(a b)$ of the specimen on the film $C$. 


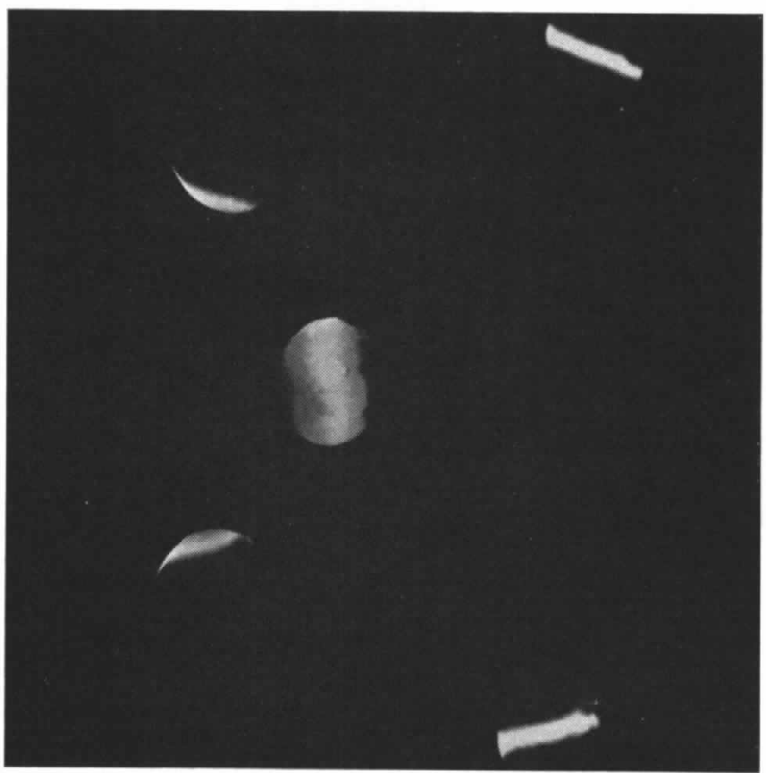

(a)

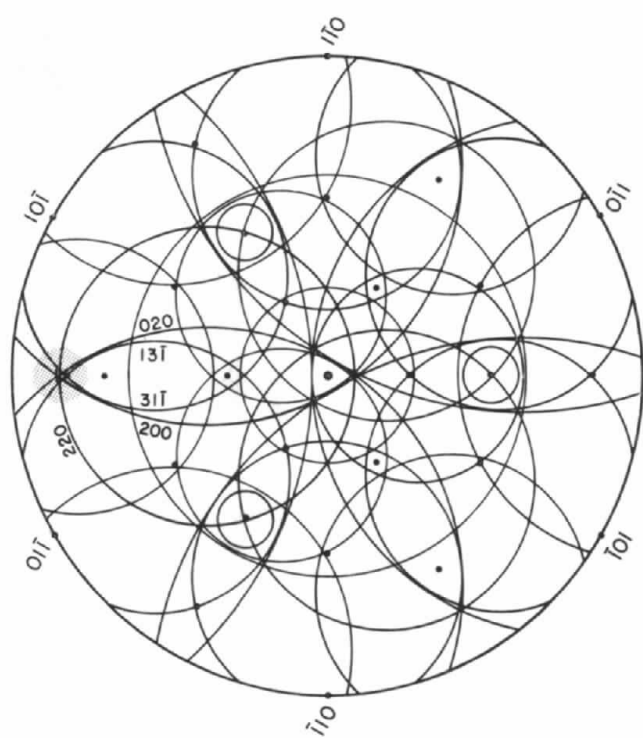

(b)

Fig. 2. (a) Five images of the (111) surface of a copper crystal produced by simultaneous diffraction of Co $K \alpha$ radiation from five sets of reflecting planes. Specimen to film distance was approximately $2 \mathrm{~cm}$. (b) Reflection loci plot showing incident beam direction (indicated by the triangle in the shaded area) which produced the five diffracted beams in $(a)$. 


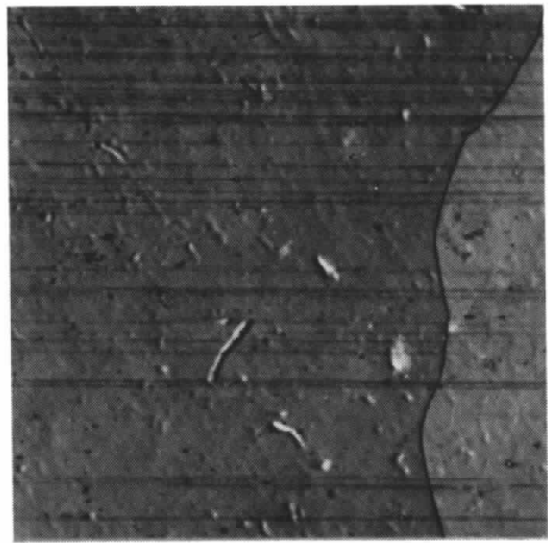

$0.02 \mathrm{~cm}$

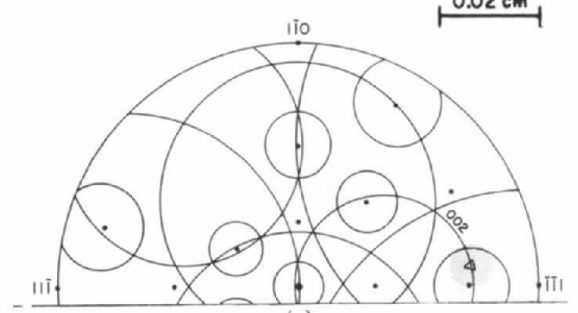

(a)

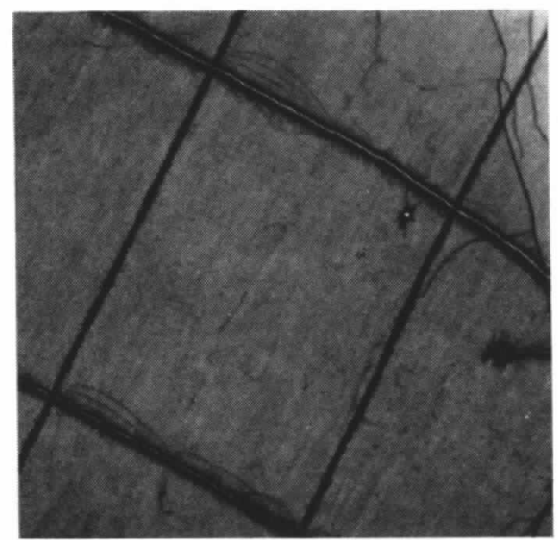

$0.02 \mathrm{~cm}$

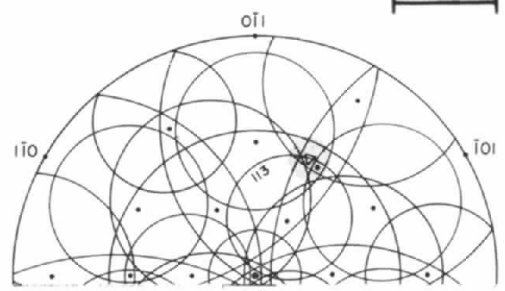

(c)

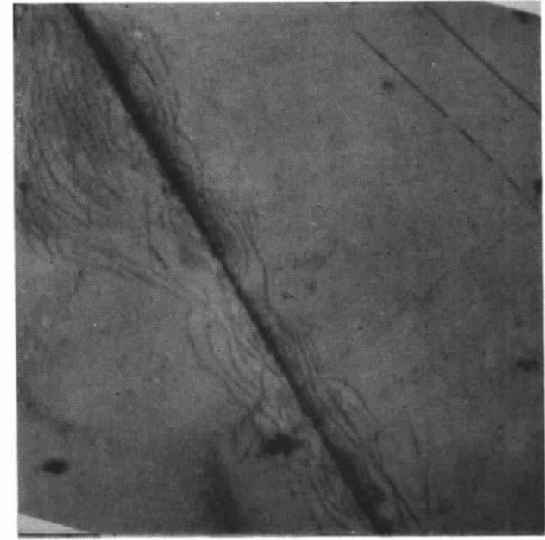

$0.02 \mathrm{~cm}$

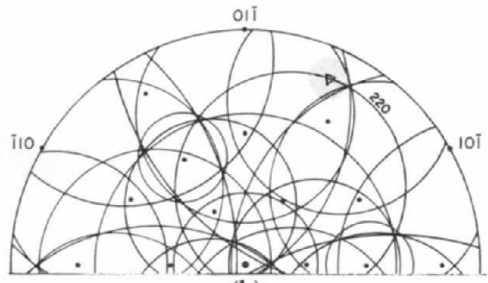

(b)

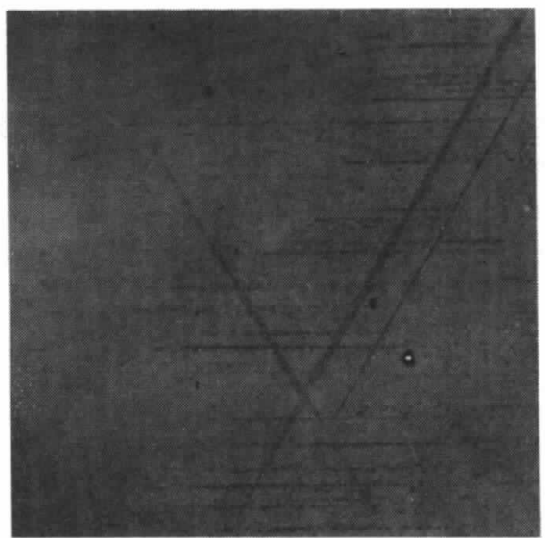

$0.02 \mathrm{~cm}$

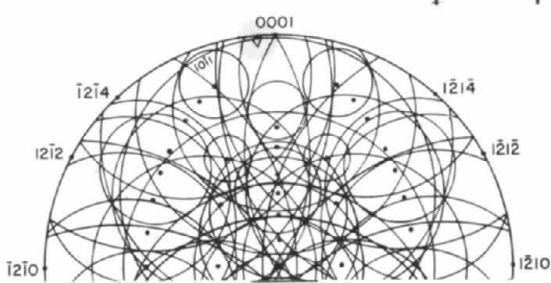

(d)

Fig. 3. B-B photographs oriented to correspond with the accompanying reflection loci plots for (a) silicon-iron, (b) aluminum, $(c)$ copper, and $(d)$ zinc. Poles of the possible reflecting planes on the interior of the plot are shown as solid circles, and the location of the incident X-ray beams for the photographs shown are indicated by a triangle in the shaded region. Kodak high resolution plates (HRP), developed in HRP developer were used. (a) Slip bands on a (112) surface of an $\mathrm{Fe}-3 \% \mathrm{Si}$ single crystal. $\mathrm{Cr}$ tube operated at $50 \mathrm{kV}$ and $7.5 \mathrm{~mA}, 0.025 \mathrm{~mm}$ vanadium foil filter, (002) reflection, $3 \mathrm{hr}$ exposure. (b) Dislocations near a scratch on the (111) surface of an aluminum single crystal. Fe tube operated at $39 \mathrm{kV}$ and $9 \mathrm{~mA}$, unfiltered (220) reflection, $2 \mathrm{hr}$ exposure. $(c)$ Dislocations with $b=(a / 2)[\overline{1} 01]$ formed by scratching the (111) surface of a copper single crystal. Substructure shows in upper right. Fe tube operated at $39 \mathrm{kV}$ and $9 \mathrm{~mA}, 0.025 \mathrm{~mm}$ Fe filter, (113) reflection, $10 \mathrm{hr}$ exposure. $(d)$ Slip bands on the $\{1 \overline{2} 12\}\langle 1 \overline{2} 13\rangle$ and the $(0001)\langle 1 \overline{2} 10\rangle$ slip systems of zinc revealed on a (10) 0 ) surface. Co tube operated at $45 \mathrm{kV}$, $6.5 \mathrm{~mA}, 0.025 \mathrm{~mm}$ Fe filter, (1011) reflection, $10 \mathrm{hr}$ exposure. 
The equation for absorption of X-rays by a filter is:

$$
R=I / I_{0}=\exp (-\mu . r),
$$

where the transmission ratio, $R$, is the ratio of transmitted intensity, $I$, to incident intensity $I_{0}, \mu$ is the linear absorption coefficient for the filter and $x$ is the path length for the X-rays to traverse the filter. In the B-B method when a filter is placed between the specimen and the film the diffracted beam which makes an angle $\alpha$ with the plane of the filter has a transmission ratio

$$
R=\exp \left(-\mu_{1} t\right) \csc \alpha,
$$

where $\mu_{1}$ is the linear absorption coefficient of the filter at the diffracted beam wavelength, and $t$ is the filter thickness. The fluorescent radiation passes through the filter over a range of angles, but that fluorescence which is most difficult to attenuate passes perpendicularly through the filter. The transmission ratio for this radiation is therefore:

$$
R^{\prime}=\exp \left(-\mu_{2} t\right)
$$

where $\mu_{2}$ is the absorption coefficient of the filter at the fluorescent radiation wavelength. The efficiency of the filter can then be expressed as the ratio of the transmission ratios of the diffracted and fluorescent beams:

$$
R^{\prime} / R=\exp \left[-t\left(\mu_{2}-\mu_{1} \csc \alpha\right)\right] \text {. }
$$

For specimens of light elements such as aluminum, $\mu_{2}$ is much greater than $\mu_{1}$ for almost all filter materials. Therefore, for the values of $\alpha$ which are most commonly used in practice, almost any filter material will yield a sufficiently large signal to noise ratio.

$K$ series fluorescence from elements such as $\mathrm{Zn}, \mathrm{Cu}$, and $\mathrm{Fe}$ however is of nearly the same energy as many of the common X-ray tube characteristic lines. Therefore, $\mu_{1}$ and $\mu_{2}$ will be significantly different only when the filter is chosen so that its $K$ absorption edge wavelength is between the wavelength of the diffracted beam and the wavelength of the fluorescence. In order for $\mu_{1}$ and $\mu_{2}$ to have the proper relationship the diffracted beam wavelength must be longer than the wavelength of the specimen fluorescent radiation. Then the fluorescence excited by the short wavelength white radiation in the tube spectrum can be sufficiently attenuated by the proper thickness of filter. It is not possible to give a definite value for $R^{\prime} / R$ which is required for the

Table 1. Compatible filters and radiation types for zinc, copper and iron specimens

$\begin{array}{ccc}\begin{array}{c}\text { Specimen } \\ \text { material }\end{array} & \begin{array}{c}\text { Radiation } \\ K \alpha \text { line }\end{array} & \text { Filter } \\ \mathrm{Zn} & \mathrm{Cu} & \mathrm{Ni} \\ & \mathrm{Co} & \mathrm{Fe} \\ & \mathrm{Fe} & \mathrm{Fe} \\ & \mathrm{Cr} & \mathrm{V} \\ \mathrm{Cu} & \mathrm{Co} & \mathrm{Fe} \\ & \mathrm{Fe} & \mathrm{Fe} \\ & \mathrm{Cr} & \mathrm{V} \\ \mathrm{Fe} & \mathrm{Cr} & \mathrm{V}\end{array}$

best results. However, for work with specimens of $\mathrm{Zn}$, $\mathrm{Cu}$, and $\mathrm{Fe}$, a value of $t\left(\mu_{2}-\mu_{1} \csc \alpha\right)>4$ was found to be adequate. Table 1 shows the radiation types which are compatible with filtering for several specimen materials, and the filter material usually used.

If specimens of heavier elements are to be examined with X-rays of wavelength between $\mathrm{Cr} K \alpha$ and $\mathrm{Cu} K \alpha$, different solutions to possible fluorescence problems would be required. The cases of Mo and $\mathrm{W}$ specimens can be considered as examples. If $\mathrm{Cu} K \alpha$ radiation is used to illuminate a Mo sample there is no filter material which will absorb the fluorescent radiation more than the diffracted beam. However, the fluorescence from the Mo can be suppressed by lowering the operating voltage of the $\mathrm{X}$-ray tube. For an operating voltage below $20 \mathrm{kV}$ no Mo $K \alpha$ fluorescence would be produced. No $K \alpha$ fluorescence would be produced from a W specimen for tube operating voltages below $70 \mathrm{kV}$. However, the W $L \alpha_{1}$ fluorescence with wavelength $1.476 \AA$ would be present. If the illuminating radiation for the $\mathrm{W}$ specimen were $\mathrm{Cu} K \alpha$ with wavelength $1.542 \AA$ the $\mathrm{W} L \alpha_{1}$ fluorescence could be filtered with a Ni filter with a $K$ absorption edge at $1.488 \AA$.

The use of a filter between the specimen and the film also helps to reduce the amount of inelastically scattered radiation which reaches the film. The incoherent radiation forms a continuous spectrum shifted slightly in energy from the X-ray tube spectrum. A single filter will not strongly attenuate all of the wavelengths in the incoherently scattered radiation. However, it has been found experimentally that the sum of the noise from fluorescence and inelastic scattering can usually be reduced to acceptable levels by the single $K$ edge filter described for attenuating the fluorescent radiation.

\section{Additional diffracted beams}

The other important source of noise is unwanted Bragg reflections of the tube $K \alpha$ characteristic which occur when the Bragg conditions are satisfied simultaneously for more than one set of reflecting planes. Fig. 2(a) shows an example in copper where the Bragg conditions were simultaneously satisfied by five sets of planes. A large specimen to film distance was used in obtaining this photograph. In the B-B method the film is placed very close to the specimen. This means that images produced by separate diffracted beams overlap and one image obscures the detail in the other. Additional diffracted beams can be avoided by properly choosing the diffraction geometry. A simple extension of a stereographic projection for the crystal in question is used to select a suitable incident beam direction. The locus of incident beam directions which satisfy the Bragg conditions is drawn on the projection for each possible reflecting plane. Fig. 3 shows several examples of these reflection loci plots. They are constructed on a stereographic representation of the crystal centered on the normal to the observation surface. A separate plot is constructed for each radiation of interest. The 
stereographic projection includes the poles of all sets of planes which can satisfy the Bragg conditions. The locus of incident directions which satisfy the Bragg conditions for a given set of planes $(h k l)$ and a given radiation is a circular cone with half apex angle $90^{\circ}-$ $\theta_{h k l}$ and the cone axis is the pole of the $(h k l)$ plane. The stereographic projection of this cone is a small circle on the Wulff net, but the center of the circle on the projection is displaced radially outward from the projection of the pole of the $(h k l)$ plane (Fig. 4). The center of this circle is easily located by the construction shown in Fig.4. First the projection is rotated until the $(h k l)$ pole lies on the equator of the Wulff net. The point $A, 90^{\circ}-\theta_{h k l}$ from the pole measured along the equator is located, and point $B, 90^{\circ}-\theta_{h k l}$ from the pole measured along a great circle of the Wulff net is located. The perpendicular bisector $\left(P P^{\prime}\right)$ of the line $A B$ is constructed. The center, $C$, of the locus circle is then the intersection of $P P^{\prime}$ with the equator. The radius of the circle is $A C$. The construction of a reflection loci plot as in Fig. 3 is accomplished by drawing the locus circles for all possible reflecting planes in the interior of the stereographic projection. Circles are not drawn for poles lying on the outer circle of the Wulff net since diffracted beams from these sets of planes go into the crystal.

A computer program was written to aid in the rapid and accurate preparation of stereographic projections and reflecting locus plots for an arbitrary crystal surface. Cartesian coordinates are calculated for the projections of the poles of interest, for the centers of the locus circles, and for points on each circle. This information is obtained in tabular form and may either be plotted by hand, or by a high speed plotter connected to the computer.

Once the reflection locus plots are prepared the incident beam directions which produce a single reflection can be easily located. Because the specimen and X-ray focal spot have dimensions greater than zero, the incident beam direction on the desired reflecting circle must have a clear angular 'window' around it. Reflecting circles for other sets of planes must not pass through this 'window' or multiple images will result. The angular dimensions of the clear 'window' required depend on the size of the projection of the specimen surface in the plane perpendicular to the incident beam, the specimen to source distance, and the size of the projection of the source focal spot in a plane perpendicular to the beam. If $d_{x}, d_{y}$ are the dimensions of the projection of the observation surface, $D$ the specimen to source distance, and $a_{x}$ and $a_{y}$ the dimension of the projection of the focal spot, the window angle is defined by

$$
\tan \frac{\beta_{i}}{2}=\begin{gathered}
d_{i}+a_{i} \\
2 D
\end{gathered}, \quad i=x, y .
$$

For the usual case where $a_{i} \ll d_{i} \ll D$ this reduces to

$$
\beta_{i} \simeq d_{i} / D \text {. }
$$

Several examples of reflection loci plots are shown in Fig. 3. Each loci plot is accompanied by a topograph taken using the reflection 'window' indicated on the plot. The incident beam direction which produced the five diffracted beams in Fig. 2(a) is indicated in the loci plot of Fig. 2(b).

\section{Other considerations}

The problems of noise discussed above place some restrictions on the radiation and incident beam directions which can be used to obtain satisfactory B-B images. However, within these limitations there remains considerable flexibility in the choice of the parameters of the B-B method. It is therefore necessary to analyse the effects of these parameters on the properties of image contrast, resolution, reflecting area, $\mathrm{X}$-ray penetration depth and exposure time, and image distortion. As a result of such an analysis it will be possible to determine the conditions which will produce the optimum B-B image for a given investigation.

Resolution, reflecting area, and image distortion for the zero layer reflection where the normals to the observation surface and the reflecting planes, and the incident and diffracted beams are all coplanar have been discussed by Newkirk (1959) and by Austerman \& Newkirk (1967). Image contrast is discussed by Bonse et al. (1967). The discussion here will therefore be limited to consideration of X-ray penetration depth and those aspects of reflecting area and penetration depth which are different in non-zero layer reflections.

\section{$X$-ray penetration depth}

The depth to which X-rays penetrate the specimen with sufficient intensity to produce contrast depends on the wavelength of the X-rays, the absorption co-



Fig. 4. Method of construction of diffraction locus circle. $A$ and $B$ are $90^{\circ}-\theta_{(h k l)}$ from the pole of the $(h k l)$ plane. $P P^{\prime}$ is the perpendicular bisector of $A B . C$ is the center of the locus circle. $O$ is the center of the stereographic projection. 
efficient of the specimen, and the angles of incidence and diffraction. An estimate of the penetration depth can be obtained by considering the attenuation of the $\mathrm{X}$-rays by true absorption alone. For true absorption the ratio of the intensity of X-rays diffracted at a distance $x$ below the observation surface to one diffracted at the surface (Fig. 5) is:

$$
I(x) / I(0)=\exp [-\mu x(\csc \varphi+\csc \gamma)] .
$$

The X-ray penetration depth may be defined as the depth $(x)$ for which this ratio has some small value. For example the penetration depth which has an $I\left(x_{p}\right) / I(0)$ value of one-tenth is

$$
x_{p}=\frac{2 \cdot 3}{\mu(\csc \varphi+\csc \gamma)} .
$$

Because this type of calculation does not consider the effect of anomalous absorption, and because it is not clear what value of $I\left(x_{p}\right) / I(0)$ should be used, it might not be quantitatively meaningful. However it is a useful basis for the comparison of penetration depths for different values of $\mu, \varphi$, and $\gamma$.

The above expression shows that $x_{p}$ decreases with $\mu$ and $(\csc \varphi+\csc \gamma)$. Since $\mu$ decreases with decreas-

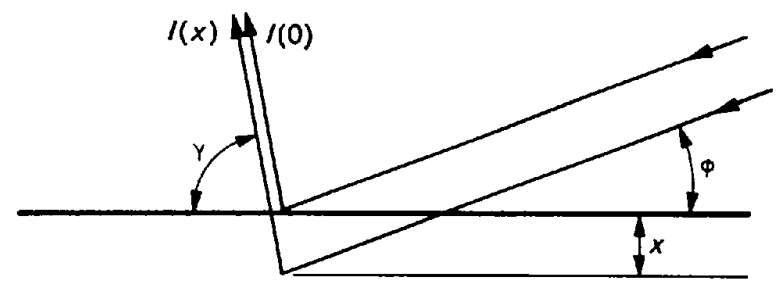

Fig. 5. The intensity of $\mathrm{X}$-rays diffracted from a distance $x$ below the surface of the specimen depends on the angles $\varphi$ and $\gamma$ and the absorption coefficient $\mu$.

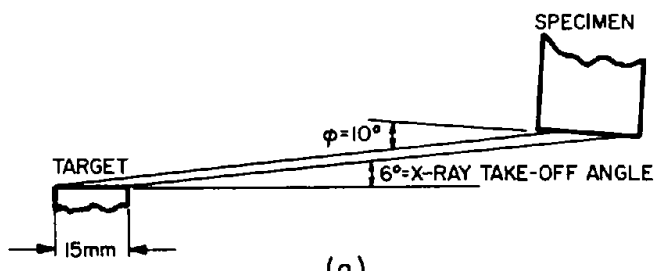

(a)

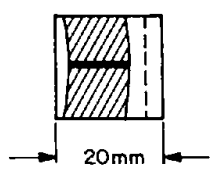

(b)

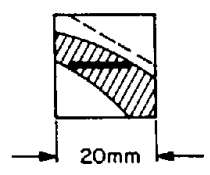

(c)
Fig. 6. The area of the observation surface which reflects depends on the effective area of the X-ray tube focal spot as projected onto the specimen surface at the take off angle $(a)$. Deviation from the zero layer condition $(b)$ changes the shape and size of the reflecting area $(c)$. Broken lines in $(b, c)$ indicate the trace of the reflecting plane. ing wavelength except for the discontinuities at absorption edges, $x_{p}$ can be maximized by using wavelengths much shorter than the $K$ edge wavelength of the specimen; or if that is impractical, by using wavelengths slightly longer than the $K$ edge wavelength.

\section{Reflecting area}

The area of the specimen surface which will reflect is bounded by the reflecting curves of the extremes of the focal spot. Therefore the extent of the reflecting area depends on the effective size and shape of the $\mathrm{X}$-ray source and the orientation of the reflecting curves. Fig. 6(a) shows the angular relationships for the projection of a $15 \times 1 \mathrm{~mm}$ focal spot onto the specimen as indicated by the solid areas in Fig. 6(b), (c). The shaded area in Fig. $6(b)$ represents the reflecting area for a zero layer reflection. The trace of the reflecting planes is shown as a dotted line in the figure. Fig. $6(c)$ shows the reflecting area for a non-zero layer reflection. The difference between zero layer and nonzero layer reflections is clearly shown in Fig. 2 where the center reflection is zero layer and the other four reflections are far from zero layer. If the observation surface of the specimen is larger in one direction than in the other, the reflecting area is maximum when the trace of the reflecting plane is parallel to the long direction; for observation of equidimensional surfaces the zero layer reflection has the maximum reflecting area.

\section{Image distortion}

Newkirk (1959) has shown that the major distortion in zero layer reflections is an elongation or foreshortening of the image in the direction parallel to the incident beam. When the film is placed parallel to the direct beam this magnification has the form:

$$
x^{\prime}=\frac{x \sin (2 \theta-\varphi)}{\sin 2 \theta^{-}},
$$

where $x$ is the true distance in the specimen surface and $x^{\prime}$ is the distance in the image.

There is also a smaller distortion in the direction perpendicular to the beam for zero layer reflections. Because points on the opposite sides of the zero layer plane diffract X-rays which are incident with opposite components normal to the zero layer plane a small magnification in the direction normal to the zero layer plane occurs. Since the film is in general not parallel to the specimen surface this magnification is different at different positions on the specimen. The effect is that the image of a square specimen is a trapezoid as in Fig. 7. This divergence is generally small and can be ignored in many applications.

For non-zero layer reflections all of the diffracted rays have a component normal to the zero layer plane. Because the film is not parallel to the specimen surface the image of a square region of the specimen will be mapped into a region which is approximately a parallelogram as shown in Fig. 7. The elongation or fore- 
shortening of the specimen in the direction parallel to the incident beam and the divergence distortion also occur for the non-zero layer image. Although the distortions cannot in general be completely eliminated they can always be calculated from a knowledge of the diffraction geometry and the orientation of the film.

\section{Utility of the reflection loci plots}

Improvement of one property of the B-B image often implies the degradation of one or more of the others. Therefore the optimum arrangement for producing a B-B image depends on the purpose of the investigation. For example, the depth of penetration increases as the incident angle increases, but the reflecting area and the area for which resolution is good decreases as the incident angle increases. Therefore, in order to observe dislocations lying fairly far below the specimen surface one would be willing to sacrifice reflecting area and resolution for increased penetration depth. However both dislocations lying close to the observation surface and slip bands are easily observed when the penetration depth is small. In these cases the increased reflecting area and resolution gained with small incident angles are more important than penetration depth.

The reflection loci plots described above contain all of the geometric information about each possible reflection for a given radiation and specimen. Consequently the loci plots have been found to be very useful for quickly evaluating the advantages and disadvantages of various possible reflections. Since the stereographic projection used is centered on the specimen surface, the angular distance from the edge of the Wulff net to the incident beam direction is the incident angle, $\varphi$. Therefore the illuminated area and the region of good resolution generally decrease in size as the incident beam direction moves toward the center of the net. Since the latter two quantities also depend on the diffracted beam direction, qualitative comparison of these properties is slightly more difficult but with practice can be done quite rapidly. Windows near the zero layer position are often desirable because increasing deviation from the zero-layer condition is associated with increasing distortion of the image and decreasing reflecting area. Penetration depth increases with deviation from the zero-layer condition, so the image distortion and decreased area may be acceptable when greater penetration depth is required.

In addition to being useful for quick qualitative comparison of various reflections, the reflection loci plots are useful for deriving the angular information needed to calculate distortions, penetration depths, and image extinctions. Since the diffracted beam direction for a given incident beam direction can easily be derived from the reflection loci plots the values of the parameters needed to calculate the distortions and penetration depths for all possible reflections are easily measured on the Wulff net. If Burgers vector directions are indicated on the projection, the reflections which can be used for image extinction of dislocations with a given Burgers vector can easily be found.

\section{Equipment}

The great advantage of the B-B method is its flexibility. Once the possible problems are understood it becomes relatively easy to obtain good results in a wide variety of situations with simple equipment. The B-B camera arrangement is shown in Figs. 9 and 10. The equipment includes the specimen holder goniometer $(A)$, standard X-ray tube with collimator slits $(B)$, and film holder $(C)$. Additional equipment used for setting up for the exposure is a fluorescent screen assembly $(D)$ and a back reflection Laue camera arrangement $(E)$.

\section{Specimen holder goniometer}

The specimen holder goniometer provides two axes of rotation and one direction of translation. One of the rotation axes is perpendicular to the observation surface of the specimen. The other axis (Bragg angle adjustment) is perpendicular to the first axis and to the incident beam direction. The axis of translation is perpendicular to the incident beam and in the plane defined by the incident beam and the normal to the observation surface. The holder is constructed so that the two axes of rotation and the incident X-ray beam intersect at a point at the center of the translation. The specimen is held so that the center of the observation surface nearly coincides with the intersection of the rotation axes. The two rotations provided are sufficient to allow the specimen to be oriented for any possible Bragg reflection.

It has been found that gear drives on these rotations are not necessary. We use a very viscous grease in the bearing surfaces of the Bragg angle adjustment so that very slow angular motions may be made. The translation provides a means for fine adjustment of the position of the specimen in the X-ray beam. A drive may be coupled to either the translation or the Bragg angle adjustment to move the specimen and film together. A scanning drive is used when the diffraction geometry is such that only a small portion of the specimen will reflect. The entire goniometer assembly may be moved on the camera track to change the specimen to X-ray source distance.

\section{Tube and collimator slits}

The X-ray tube used was either a General Electric CA8S or Machlett A2. The X-ray tube is fitted with a special shutter assembly which holds a collimator tube

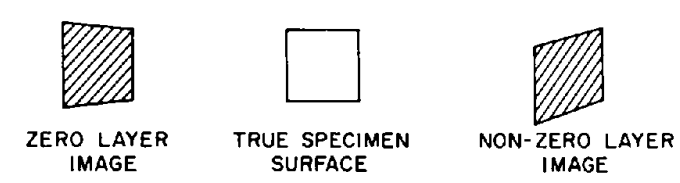

Fig. 7. Image distortion caused by the component of the diffracted beam perpendicular to the zero-layer plane. 

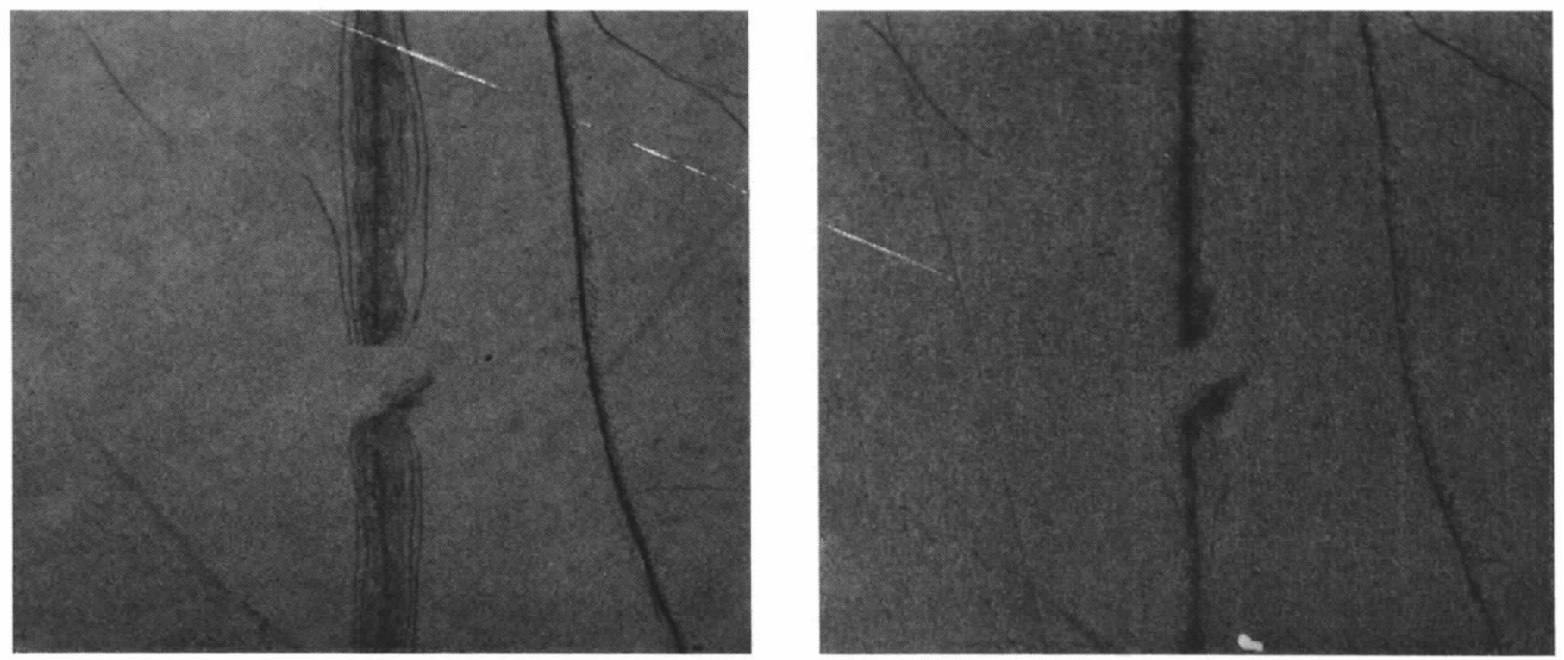

(a)

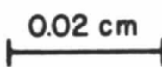

(b)

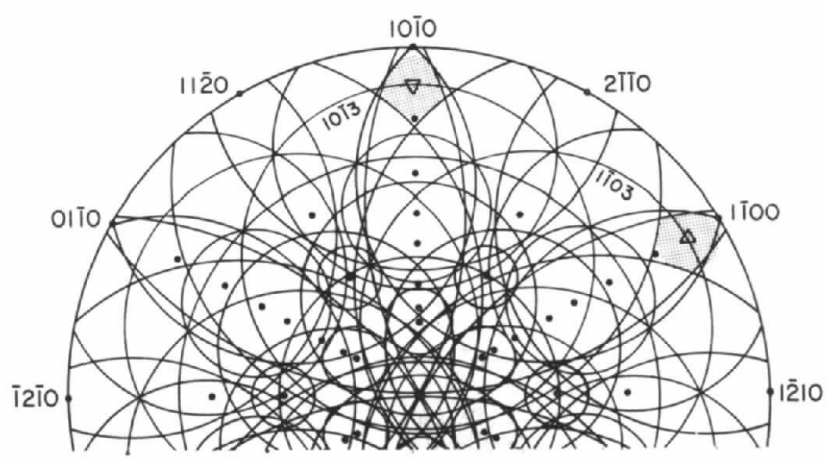

(c)

Fig. 8. B-B contrast at dislocations with $\mathbf{b}=\frac{1}{3}[1 \overline{2} 10]$ parallel to a (0001) surface of a zinc single crystal. The dislocations were produced by scratching the crystal surface. The photographs were taken with a General Electric CA $8 \mathrm{~S}$ Co tube operated at $45 \mathrm{kV}, 6.5 \mathrm{~mA}$, using a $0.025 \mathrm{~mm}$ thick Fe foil filter with an exposure time of $8 \mathrm{hr}$. (a) Reflection from the (1T⿱1003) planes for which $\mathbf{b} \cdot \mathbf{n} \neq 0$. (b) Reflection from the (1013) planes for which $\mathbf{b} \cdot \mathbf{n}=0$. (c) The reflection loci plot indicating the incident beam directions for the pictures $(a)$ and $(b)$, and the orientations of the crystal surface in the photographs. 


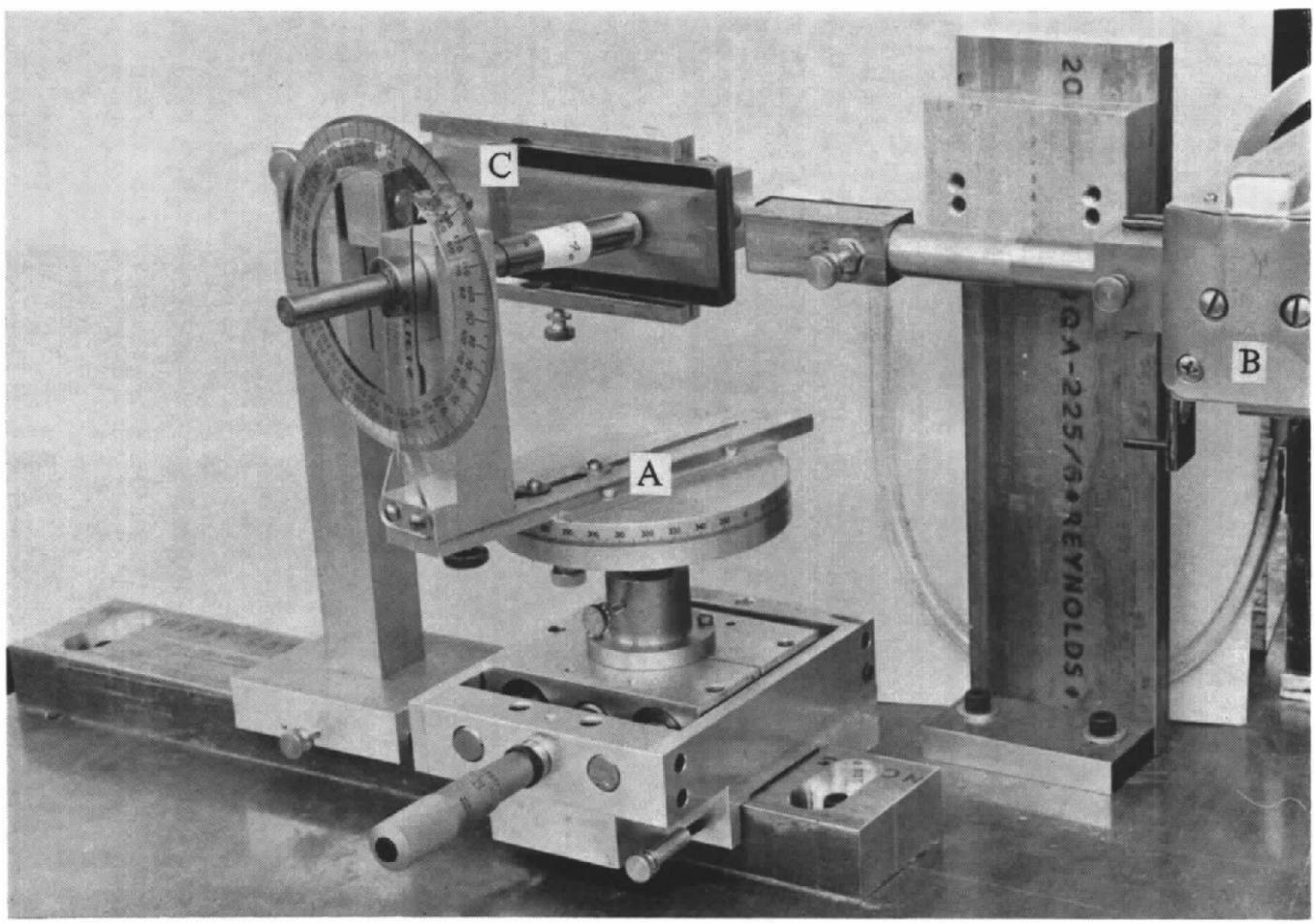

Fig.9. X-ray apparatus used in the B-B method. $A$, specimen holder goniometer. $B$, General Electric CA 8S X-ray tube with collimator slits. $C$, Film holder.

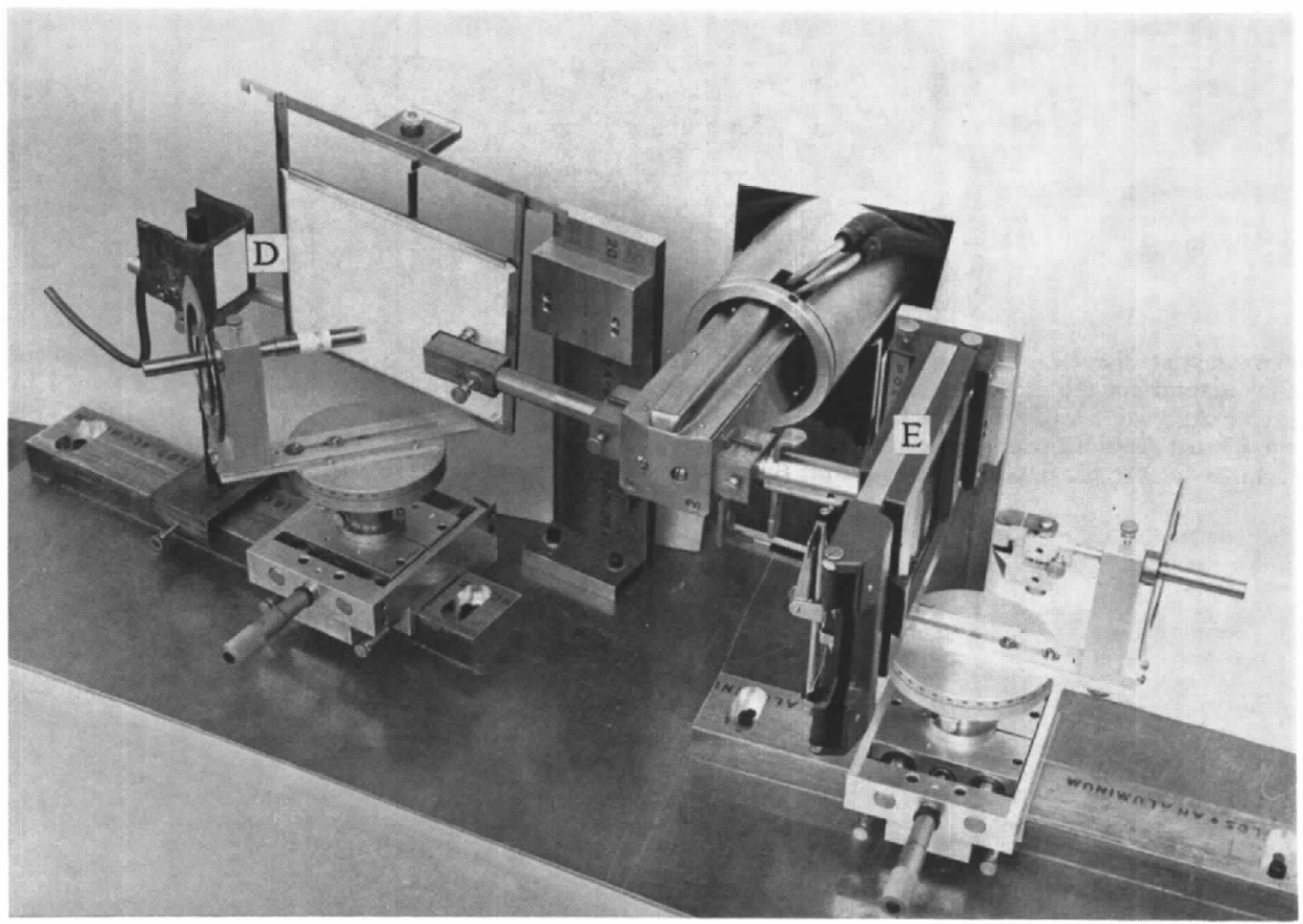

Fig. 10. Equipment used to set up B-B apparatus. $D$, Fluorescent screen assembly. $E$, Back reflection Laue camera. 


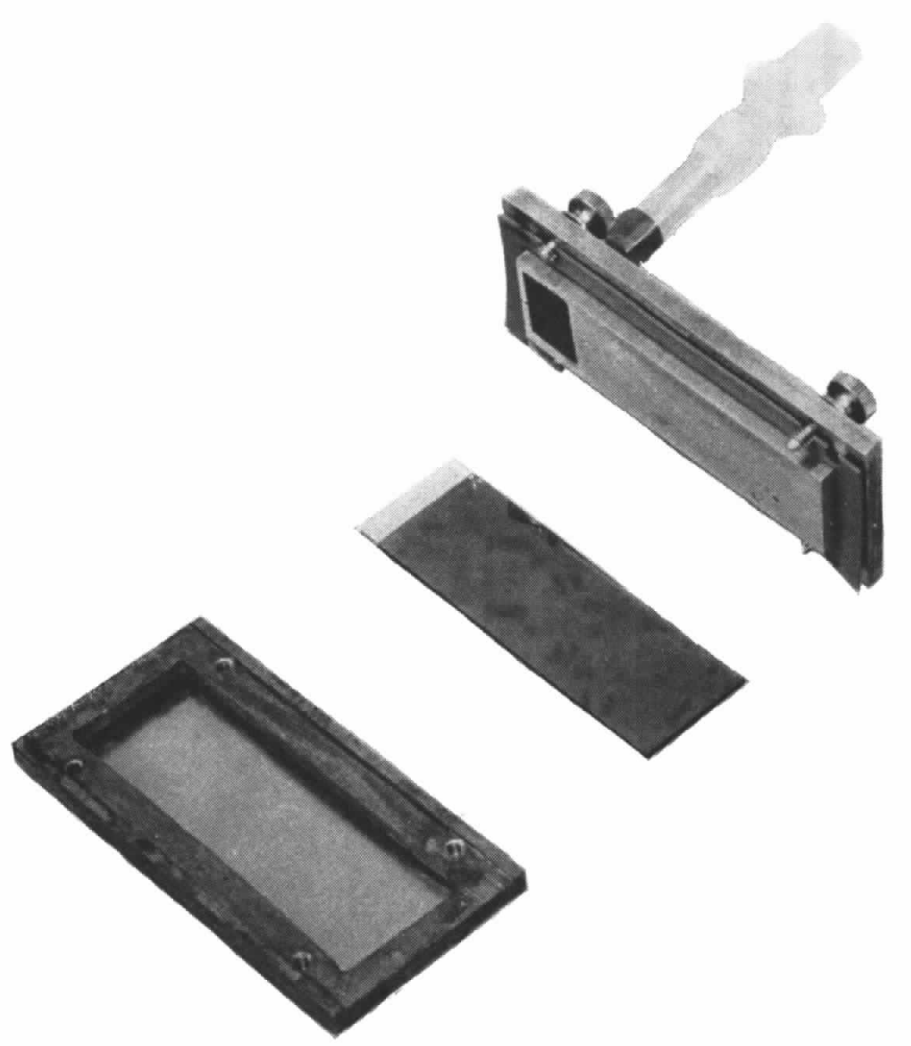

Fig. 11. Vacuum cassette. 


\section{$0.02 \mathrm{~cm}$}

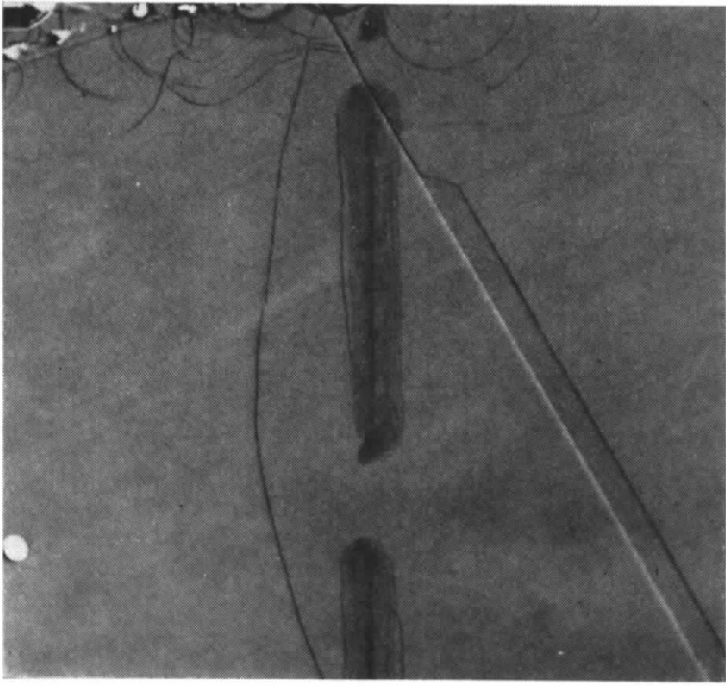

(a)

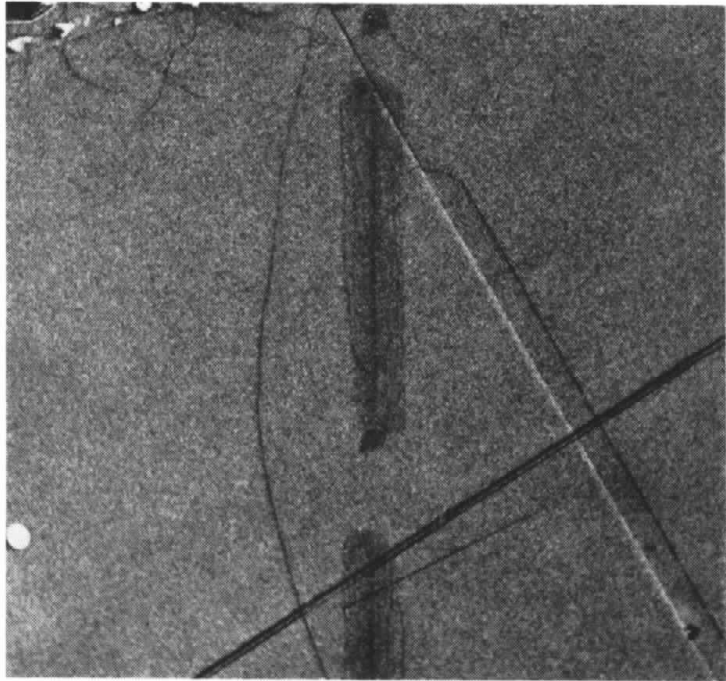

(b)

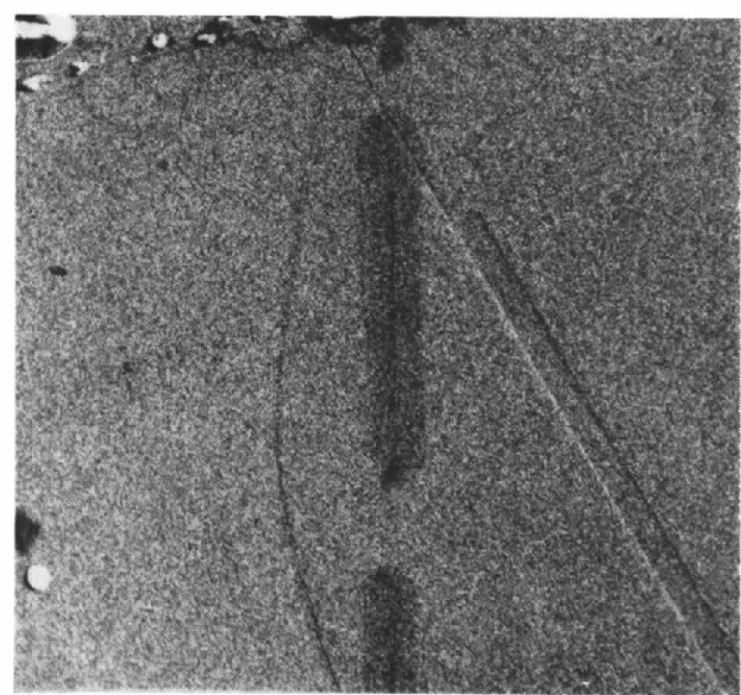

(c)

Fig. 12. Comparison of dislocation images on (a) HRP, $(b)$ AR. 10, (c) type R emulsions. The dislocations were produced by scratching a (0001) surface of a zinc crystal. The Burgers vectors are $\frac{1}{3}[1 \overline{2} 10]$. The reflecting plane is (1103). 
and slits. The axis of the collimator tube makes an angle of about $6^{\circ}$ with the plane of the X-ray focal spot (X-ray take-off angle). One side of the slit is movable to adjust the slit width. The position of the specimen relative to the slit is adjusted by means of the specimen holder translation described above. It should be emphasized here that the slits are used only to prevent the unused portions of the incident beam from fogging the film or causing a hazard to the operator. The B-B method does not require a collimated X-ray beam.

\section{Film holder}

The film cassette is shown in Fig. 11. It is designed so that no part of the cassette interferes with positioning of the film close to the specimen. The front of the cassette is either a steel shim stock foil $0.025 \mathrm{~mm}$ thick or an opaque polyethelene sheet $0.125 \mathrm{~mm}$ thick. The former is used when an Fe filter is appropriate. When other filter materials are desired a foil of the filter material is placed inside the polyethelene covered cassette. The tube on the cassette is attached to a vacuum pump so that the front of the cassette is held tightly against the film. This makes it possible to position the film very close to the specimen.

The clamp which holds the cassette is provided with an axis of translation and an axis of rotation. The film cassette is moved toward the specimen by a lead screw. This means of positioning the film minimizes the possibility of hitting the specimen with the film in the process of placing it close to the specimen. The rotation is provided because in some instances the specimen-tofilm distance can be decreased by rotating the plane of the film.

The cassette clamp is usually attached to the camera track. When scanning is necessary, the cassette clamp is attached to the moving portion of the specimen holder.

\section{Fluorescent screen assembly}

The fluorescent screen assembly replaces the film holder for the setting-up procedure. The assembly consists of a support and two fluorescent screens, one behind the specimen to intercept the direct beam, and one positioned to intercept the diffracted beam. The screens are backed by lead to stop the X-rays. The specimen is positioned in the beam and the slit width is set by observing the shadow of the specimen on the rear screen. The angular adjustments of the goniometer are made so as to maximize the intensity of the diffracted beam observed on the fluorescent screen.

\section{Laue camera}

It is not possible to take Laue patterns of the specimen with a standard camera arrangement after it is mounted on the goniometer because the goniometer assembly is too large. Therefore the special camera support was provided so that in-place Laue patterns can be obtained when they are needed. When the specimen surface is a known crystallographic plane and the reflection loci plot for that surface has been made, the desired reflection can be easily found by observing the reflections which fall on the fluorescent screen while angular adjustments of the specimen are made. The Laue camera is not needed in this case.

\section{Obsertation surface preparation}

Special care must be taken during the preparation of the observation surface of the crystal. The surface must be smooth on the scale of the X-ray penetration depth if any significant length of dislocations is to be observed. The thickness of the oxide layer or other surface contamination must also be small compared with the X-ray penetration depth. Furthermore, the dislocation density near the surface must be less than about $10^{6} \mathrm{~cm}^{-2}$ or the dislocation images will overlap producing a uniform darkening of the photographic plate. This last requirement rules out the use of any abrasive material for final preparation of this surface. We have found acid or electrolytic machining techniques to be the most useful. A B-B photograph of a well-annealed carefully prepared metal specimen will commonly reveal very few if any dislocations which lie parallel to the surface (except for dislocations in substructure). We have found that dislocations can best be introduced into a well-annealed specimen by making a light scratch on the observation surface. On $\mathrm{Zn}, \mathrm{Cu}$ and $\mathrm{Al}$ specimens, scratches are usually made with an $\mathrm{Al}_{2} \mathrm{O}_{3}$ whisker loaded with $50-500 \mathrm{mg}$. Scratches on $\mathrm{Fe}-3 \% \mathrm{Si}$ are made with a diamond stylus with a load of about $5 \mathrm{~g}$.

\section{Films}

Since the B-B method produces unmagnified photographs of a specimen it is often useful to magnify the photographs $50 \times$ or more. Therefore a fine grained film is desirable. We have found Kodak High Resolution Plates (HRP) to have ample resolution for all our applications. The main drawback of these plates is the long exposure times they require. We usually develop these plates about twice as long as recommended by Kodak to decrease exposure times and increase contrast. We have also found Kodak Fine Grain Autoradiographic Stripping Plates, type AR.10 to be useful. The resolution of these plates is lower than that of the HRP but it is sufficient for many applications. AR.10 Plates require about $1 / 15$ the exposure of the HRP. These plates however are rather inconvenient in that the emulsion must be transferred to a new back. Rekordak Micro-File Film type 5455 (Eastman Kodak Co.) has about the same speed as the stripping plates and yields a slightly lower quality image. Kodak Industrial X-Ray Film, type R, single coated, is very useful for obtaining very fast preliminary photographs of a specimen. This film requires about $1 / 80$ the exposure of an HRP. Fig. 12 shows a comparison of the HRP, AR.10, and type R emulsions. We have not tried any Ilford or Gevaert emulsions, so we cannot report on their relative merits. 


\section{Summary}

We have attempted to give an orderly presentation of the problems to be solved and the considerations to be made when the B-B technique is applied to any given situation. Perhaps a brief summary of the various steps is in order.

The choice of an observation surface is usually dictated by considerations not directly connected with the B-B technique. For example, to see dislocations in a material that does not form slip bands the observation surface must be nearly parallel to a slip plane. A choice of radiation must then be made. If the material is of low atomic number, the wavelength that gives the best diffraction geometry can be selected. If the material is of higher atomic number like $\mathrm{Zn}, \mathrm{Cu}$ or $\mathrm{Fe}$ then the choice must be made with a consideration of specimen fluorescence and the possibility of filtration. An observation surface and a radiation (or perhaps several possible radiations) having been selected, the reflection loci should be plotted. The loci can be used in the selection of the radiation (if there is a choice), the reflecting plane and the incident beam direction. The possible incident beam directions are those for which a clear angular window exists on the reflection loci plot through which only one reflecting circle passes. The final choice of the incident beam direction must be based on considerations of contrast, penetration depth, illuminated area, resolution and image distortion.

We feel that part of the beauty of the B-B method is the simplicity of the equipment required. A well collimated X-ray beam is neither necessary nor desirable. Since the X-ray beam is not collimated, the Bragg angle adjustments are not critical and can be best made by rotating a smooth, viscous bearing by hand while watching the image on a fluorescent screen.

Care must be exercised in the preparation of the observation surface. If the surface is abraded there is little chance of seeing individual dislocations due to the high dislocation density.
The choice of a film can be made on the basis of the resolution required. An ultra fine grain film like Kodak High Resolution Plates may be required to image individual dislocations reliably. Since fine grained films require long exposure times, one might select a more coarse grained film which will give less resolution, but will require a significantly shorter exposure.

The work reported herein was sponsored by the U.S. Atomic Energy Commission under Contract AT(04-3)-473. Dr R.C.Blish II, and Mr Jeffrey A. Gorman kindly supplied the B-B plates showing slip bands in $\mathrm{Zn}$ and dislocations in Al. Discussions with Professor D.S. Wood during the initial stages of this study were very helpful. A.P.L. Turner wishes to express his thanks to the Fannie and John Hertz Foundation for fellowship support during the course of this work.

\section{References}

Armstrong, R. W. (1965). Phys. Stat. Sol. 11, 355.

Austerman, S. B., Newkirk, J. B. \& SMith, D. K. (1965). J. Appl. Phys. 36, 3815.

Austerman, S. B. \& Newkirk, J. B. (1967). Advances in $X$-ray Analysis, p. 124. New York: Plenum Press.

BONSE, U. K., HART, M. \& NewKIRK, J. B. (1967). Advances in X-ray Analysis. New York: Plenum Press.

Lommel, J. M. \& KRonberg, M. L. (1962). Direct Observations of Imperfections in Crystals, p.543. New York: Interscience Publishers.

NeWKIRK, J. B. (1959). Trans. AIME, 215, 583.

POPE, D. P., VReEland, T., JR. \& WOOD, D. S. (1967). $J$. Appl. Phys. 38, 4011.

RoberTs, J. M. \& Gow, K. V. (1958). Trans. AIME, 212 , 648.

Robinson, W. H. \& Levinstein, H. J. (1962). Direct Observations of Imperfections in Crystals, p.561. New York: Interscience Publishers.

Schultz, J. M. \& Armstrong, R. W. (1964). Phil. Mag. $10,497$.

WeBB, W. W. (1962). Direct Observations of Imperfections in Crystals, p. 29. New York: Interscience Publishers. 